PROCEEDINGS OF THE

AMERICAN MATHEMATICAL SOCIETY

Volume 125, Number 10, October 1997, Pages 3003-3009

S 0002-9939(97)04027-6

\title{
MILNOR FIBRATIONS OF LATTICE-ISOTOPIC ARRANGEMENTS
}

\author{
RICHARD RANDELL
}

(Communicated by Ronald A. Fintushel)

\begin{abstract}
We show that the associated Milnor fibrations are equivalent in a smooth family of arrangements with constant intersection lattice.
\end{abstract}

\section{INTRODUCTION}

We consider arrangements $\mathcal{A}=\left\{H_{1}, \ldots, H_{n}\right\}$ of linear codimension one subspaces of an $\ell$-dimensional complex vector space $V$, with complement $M=V-\cup H_{i}$. In [4] it was shown that the lattice of intersections of subsets of the hyperplanes determines the cohomology ring of the complement. In [5] we showed that the diffeomorphism type of the complement is constant in a smooth one-parameter family of arrangements in which the lattice of intersections is unchanged (a "lattice isotopy," defined in Section 2.) Each subspace $H_{i}$ is defined as the zero locus of a linear form $\alpha_{i}$, defined up to a constant factor, and we may consider $M$ as the complement of the divisor associated to the product $Q=\prod \alpha_{i}$.

There is a Milnor fibration [3]

$$
Q^{-1}(1)=F \rightarrow M \rightarrow \mathbb{C}-\{0\},
$$

where the right-hand map is simply $Q$. Note that $Q$ is defined up to a constant, but that by the homogeneity of $Q$, the fiber bundle one gets is independent of the choice of constant. We call this the Milnor fibration associated to the arrangement.

In this paper we will apply Thom's second isotopy lemma to prove that the isomorphism type of the Milnor fibration is constant in a smooth one-parameter family of arrangements in which the intersection lattice is constant. This is a natural extension of the result of [5].

A question of considerable interest in the theory of arrangements is whether or not the intersection lattice determines the topology of the complement. The main result of this paper shows that fine invariants of the situation, such as the Milnor fiber, are determined by the intersection lattice, within smooth families.

In Section 2 we give the relevant definitions and make some basic observations. In particular we give a precise statement of Thom's second isotopy lemma. In Section 3 we give the proof of the main result. This involves setting up appropriate spaces and verifying that the hypotheses of Thom's lemma hold. As noted, these results focus interest on the Milnor fiber.

Received by the editors April 8, 1996.

1991 Mathematics Subject Classification. Primary 52B30; Secondary 57R52.

(C)1997 American Mathematical Society 


\section{DEFINITIONS AND NECESSARY BACKGROUND}

Our theorem for lattice-isotopic arrangements follows from the second isotopy lemma of R. Thom (as proved by J. Mather [2]). This result, which allows one to conclude that a family of maps is "locally trivial", is perhaps not as accessible as it would merit, so we give here the relevant definitions and a precise statement.

Suppose that $f: M \rightarrow S$ is a smooth map of smooth manifolds, and let $X$ be a closed subset of $M$. A stratification of $X$ is a partition of $X$ into a finite collection of submanifolds (called strata $X_{\alpha}$ ) of $M$ so that the following frontier condition holds:

$$
\text { if } X_{\beta} \cap \bar{X}_{\alpha} \neq \emptyset, \text { then } X_{\beta} \subset \bar{X}_{\alpha} \text {. }
$$

In [7] H. Whitney introduced two extra conditions on stratifications, which he called conditions a) and b):

a) Whenever $X_{\beta} \subset \bar{X}_{\alpha}$ and $x_{i} \in X_{\alpha}$ is a sequence converging to $y \in X_{\beta}$, with $\left.T X_{\alpha}\right|_{x_{i}} \rightarrow T \subset T M_{y}$, then $\left.T X_{\beta}\right|_{y} \subset T$.

b) For every $y \in X_{\beta}$, direction $\ell$, and plane $T$ of dimension $d_{\alpha}=\operatorname{dim} X_{\alpha}$, if there are sequences $x_{i} \in X_{\alpha}, y_{i} \in X_{\beta}$ so that $x_{i} \rightarrow y, y_{i} \rightarrow y, x_{i}-y_{i} \rightarrow \ell$ and $T_{x_{i}} X_{\alpha} \rightarrow T$, then $\ell \in T$.

A stratification is called a Whitney stratification if it satisfies condition b) for all $y \in X_{\beta} \subset \bar{X}_{\alpha}$. We note that condition b) actually implies condition a). See [1] or [7] for further discussion of Whitney stratifications.

The smooth map $f: M \rightarrow S$ is said to be stratified provided that one has Whitney stratifications $\Sigma=\left\{X_{\alpha}\right\}$ and $\sigma=\left\{S_{\alpha}\right\}$ of $X$ and $S$ respectively, that $f$ maps stratum into stratum, and that $f \mid X_{\alpha}$ is a submersion for every stratum $X_{\alpha}$.

Thom's first isotopy lemma [2, p.62] says that a proper smooth stratified map is the projection of a smooth fiber bundle. This result was used in [5, Theorem 1] to show that lattice-isotopic arrangements are topologically isomorphic.

We next recall some definitions from [5]. For any arrangement, one has the associated lattice, which consists of the various intersections of collections of hyperplanes $H_{i}$, ordered by reverse inclusion. Suppose that $I$ is an open interval of real numbers, containing 0 and 1.

Definition 1. A smooth 1-parameter family of arrangements $\mathcal{A}=\left\{A_{t}\right\}$ is a finite collection $\left\{H_{i t}\right\}, i=1,2, \ldots, n$, of subspaces for each $t \in I$ so that $H_{i t}$ is the locus in $V \times\{t\} \subset V \times I$ of an equation linear in the coordinates of $V$ with coefficients smooth functions of $t$. Thus, the defining equations have the form

$$
\alpha_{i}(z, t)=\alpha_{i 1}(t) z_{1}+\alpha_{i 2}(t) z_{2}+\ldots+\alpha_{i \ell}(t) z_{\ell},
$$

where the $\alpha_{i j}: I \rightarrow \mathbb{C}$ are smooth functions.

A one-parameter family gives arrangements $\mathcal{A}_{t}$ for any $t \in I$ in the obvious way.

Definition 2. Arrangements $\mathcal{A}_{0}=\left\{H_{1}, H_{2}, \ldots, H_{n}\right\}$ and $\mathcal{A}_{1}=\left\{G_{1}, G_{2}, \ldots, G_{n}\right\}$ have the same lattice if for all $J \subset\{1,2, \ldots, n\}$,

$$
\operatorname{dim} \cap_{i \in J} H_{i}=\operatorname{dim} \cap_{i \in J} G_{i} .
$$

Definition 3. A one-parameter family $\left\{\mathcal{A}_{t}\right\}$ is a lattice isotopy provided that for any $t_{1}, t_{2}$ the arrangements $\mathcal{A}_{t_{1}}$ and $\mathcal{A}_{t_{2}}$ have the same lattice. 
Let $Q_{i t}$ be the products of the linear forms defining the hyperplanes $H_{i t}$ and set

$$
Q_{t}(z)=\prod_{i=1}^{n} \alpha_{i}(z, t)
$$

Then there is the map $Q: \mathbb{C}^{\ell} \times \mathbb{R} \rightarrow \mathbb{C} \times \mathbb{R}$ defined by $Q(z, t)=\left(Q_{t}(z), t\right)$. For a fixed $t \in I$, one has the Milnor fibration $Q_{t}$ from the complement of the hyperplanes of the arrangement $\mathcal{A}_{t}$ into $\mathbb{C}^{*}=\mathbb{C}-\mathbf{0}$, with Milnor fiber $F_{t}=Q_{t}^{-1}(1)$. The main result of this note is that this fibration is independent of $t$.

To make this notion of independence of $t$ precise we adopt the following definitions of $[2, \mathrm{p} .63]$.

Suppose we are given the following diagram of spaces and mappings:

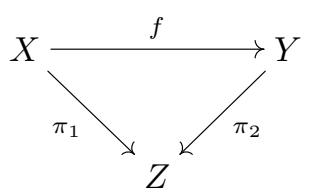

We say that $f$ is trivial over $Z$ provided that there are spaces $X_{0}$ and $Y_{0}$ and a mapping $f_{0}: X_{0} \rightarrow Y_{0}$ with homeomorphisms $h_{1}: X \rightarrow X_{0} \times Z, h_{2}: Y \rightarrow Y_{0} \times Z$ so that the following diagram commutes:

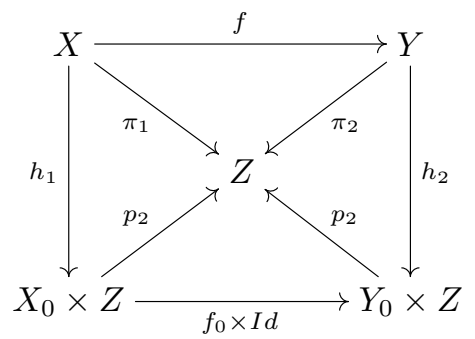

$f$ is locally trivial over $Z$ if for all $z \in Z$ there is a neighborhood $U$ of $z \in Z$ so that in the diagram

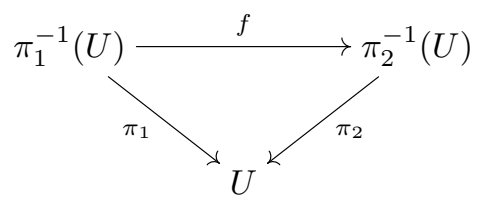

we have $f$ trivial over $U$.

Our next goal is to define a class of mappings which are locally trivial. Suppose $g: M^{\prime} \rightarrow M$ is a smooth mapping, and suppose that $S^{\prime}$ and $S$ are closed, Whitneystratified subsets of $M^{\prime}$ and $M$ respectively, with $g\left(S^{\prime}\right) \subseteq S$.

Suppose that $X_{\alpha}$ and $X_{\beta}$ are strata of the Whitney stratification of $S^{\prime}$, with $y \in X_{\beta}$. Suppose that $g \mid X_{\alpha}$ and $g \mid X_{\beta}$ are of constant rank. We then say that $\left(X_{\alpha}, X_{\beta}\right)$ satisfies condition $a_{g}$ at $y$ provided that the following holds:

Let $x_{i}$ be any sequence of points in $X_{\alpha}$ converging to $y$. Suppose that the sequence of planes $\operatorname{ker}\left(d\left(g \mid X_{\alpha}\right)_{x_{i}}\right) \subset T M_{x_{i}}^{\prime}$ converges to a plane $\tau \subseteq T M_{y}^{\prime}$ in the appropriate Grassmannian bundle. Then $\operatorname{ker}\left(d\left(g \mid X_{\beta}\right)_{y}\right) \subset \tau$.

We say that $\left(X_{\alpha}, X_{\beta}\right)$ satisfies condition $a_{g}$ if it satisfies condition $a_{g}$ at every point $y$ of $X_{\beta}$. 
Suppose we then have a diagram

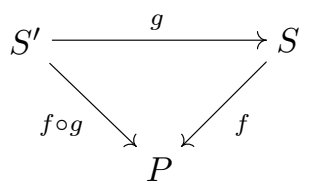

Definition 4. $g$ is a Thom mapping over $P$ if and only if the following four conditions are satisfied:

(a) $g \mid S^{\prime}$ and $f \mid S$ are proper.

(b) For each stratum $Y$ of the stratification of $S, f \mid Y$ is a submersion.

(c) For each stratum $X_{\alpha}$ of $S^{\prime}, g\left(X_{\alpha}\right)$ lies in a stratum $Y$ of $S$, and $g: X_{\alpha} \rightarrow Y$ is a submersion.

(d) Any pair $\left(X_{\alpha}, X_{\beta}\right)$ of strata of $S^{\prime}$ satisfies condition $a_{g}$.

Theorem (Thom's second isotopy lemma, [2, p. 66]). If $g$ is a Thom mapping over $P$, then $g$ is locally trivial over $P$.

\section{MAIN RESULT}

Theorem. Lattice-isotopic arrangements have smoothly equivalent Milnor fibrations.

In other words, we have a commuting diagram

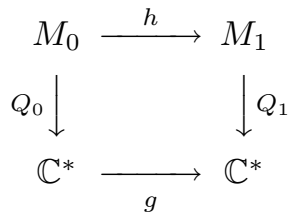

where $h$ and $g$ are diffeomorphisms.

Proof. We wish to obtain a set-up so that we can apply the second isotopy lemma. One difficulty is that the map $Q_{t}: \mathbb{C}^{\ell} \rightarrow \mathbb{C}$ is not proper. Thus we consider the restriction of $Q_{t}$ to the unit ball of $\mathbb{C}^{\ell}$, obtaining proper maps $Q_{t}: D_{t}^{2 \ell} \rightarrow \mathbb{C}$ and $Q: D^{2 \ell} \times I \rightarrow \mathbb{C} \times I$. Consider the diagram

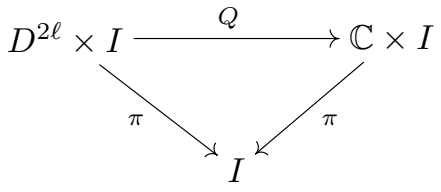

with $\pi$ denoting the projections. Recall $I$ is an open interval containing 0 and 1 .

We wish to show that $Q$ is trivial over $I$. We stratify $\mathbb{C} \times I$ by $\{(\mathbb{C}-\{0\}) \times I$, $\{0\} \times I\}$, clearly a Whitney stratification.

We use the arrangement to stratify $D^{2 \ell} \times I$. For any fixed arrangement $\mathcal{A}$, there is a natural stratification obtained by taking as strata the elements of the intersection lattice with all other lattice elements removed. That is, any element $X$ of the lattice determines an arrangement $\mathcal{A}^{X}$ in $X$, whose hyperplanes are all $H_{i} \cap X$ for hyperplanes $H_{i}$ of the original arrangement which do not contain $X$. Then one obtains a stratification of $\mathbb{C}^{\ell}$ by taking as strata all sets $M^{X}=X-\cup\left(H_{i} \cap X\right)$ together with the top-dimensional stratum $M$. By taking intersections of these strata with $\operatorname{Int}\left(D^{2 \ell}\right)$ and $\partial D^{2 \ell}$ one obtains a stratification of $D^{2 \ell}$. 
Finally, notice that since in a lattice isotopy of arrangements we require unchanging intersection lattice, we may take $V_{X}=\cup_{t} M_{t}^{X}$ intersected with $\operatorname{int}\left(D^{2 \ell}\right) \times I$ and with $\partial\left(D^{2 \ell}\right) \times I$ to form a stratification of $D^{2 \ell} \times I$.

As in [5, p.557], this forms a Whitney stratification of $D^{2 \ell} \times I$. (The proof uses the additional and obvious fact that for any lattice element $X$, the manifold $\cup_{t} X_{t}$ intersects $\partial D^{2 \ell} \times I$ transversely.)

We now check the conditions of Thom's second isotopy lemma for the diagram

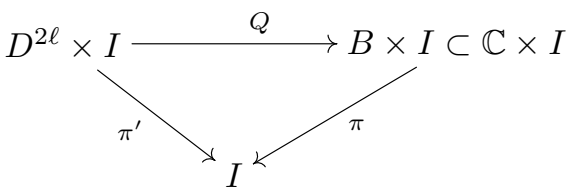

where $B$ is a sufficiently large closed disk in $\mathbb{C}$, chosen so that $Q\left(D^{2 \ell} \times I\right) \subset$ $(\operatorname{Int} B) \times I$

(a) $\left.Q\right|_{D^{2 \ell} \times I}$ and $\left.\pi\right|_{B \times I}$ are proper, because $Q$ and $B$ are compact.

(b) We stratify $B \times I$ with three strata; $\partial B \times I$, (Int $B-\{0\}) \times I$, and $\{0\} \times I$. This is clearly a Whitney stratification. Clearly $\pi$, as projection to the $I$ factor, is a submersion on these strata.

(c) We need to check that $Q$ maps strata to strata submersively. The closed disk $B$ was chosen so that $Q$ maps into $\operatorname{Int}(B) \times I$. Thus each stratum of $D^{2 \ell} \times I$ maps either into (Int $B-\{0\}) \times I$, or into $\{0\} \times I$. Strata containing points lying on hyperplanes map to $\{0\} \times I$, and since the lattice is constant throughout the isotropy, the restriction of $Q$ to such strata is a submersion.

Consider a point $\left(v, t_{0}\right)$ on the top-dimensional stratum $X$. That is, $v$ lies on no hyperplane of the arrangement $\mathcal{A}_{t_{0}}$. Clearly the same holds for $(v, t)$ for all $t$ in some small open interval $I^{\prime} \subset I$, centered at $t_{0}$. Thus we may find an open set $V \times I^{\prime}$, containing $(v, t)$, with $V \times I^{\prime} \subset X$. Consider $\left.Q\right|_{V \times I^{\prime}}: V \times I^{\prime} \rightarrow(\operatorname{Int} B-\{0\}) \times I$. The Jacobian of this map has the form

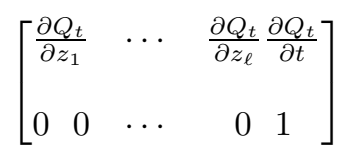

and hence is a submersion on $V \times I^{\prime}$, since $\frac{\partial Q_{t}}{\partial z_{1}}=\frac{\partial Q_{t}}{\partial z_{2}}=\cdots=\frac{\partial Q_{t}}{\partial z_{\ell}}=0$ at some $(v, t)$ implies $Q_{t}(v)=0$. (That is, 0 is the only critical value of $Q_{t}$, for all $t \in I$.)

(d) Finally, and most crucially, we need to verify that any pair $\left(X_{\alpha}, X_{\beta}\right)$ of strata of $D^{2 \ell} \times I$ satisfy the condition $A_{Q}$. This condition only has content in the case that there is a sequence $\left\{x_{i}\right\} \subset X_{\alpha}$ converging to $y \in X_{\beta}$. Our strata for $D^{2 \ell} \times I$ are

$$
\begin{aligned}
& M_{i}=\left(\underset{t}{\cup} M_{t}\right) \cap\left(\operatorname{int}\left(D^{2 \ell}\right) \times I\right), \\
& M_{b}=\left(\cup_{t} M_{t}\right) \cap\left(\left(\partial D^{2 \ell}\right) \times I\right),
\end{aligned}
$$

and for any $X$ in the (common) lattice ( $X$ not minimal)

$$
\begin{aligned}
& M_{i}^{X}=\left(\cup_{t} M_{t}^{X} \cap\left(\operatorname{int}\left(D^{2 \ell}\right) \times I\right),\right. \\
& M_{b}^{X}=\left(\cup_{t} M_{t}\right) \cap\left(\left(\partial D^{2 \ell}\right) \times I\right) .
\end{aligned}
$$


Here of course the subscripts " $i$ " and " $b$ " refer to interior and boundary respectively. Actually, $M_{i}$ and $M_{b}$ correspond to the lowest lattice element, the intersection of the empty set of hyperplanes.

Thus we must verify non-vacuous cases (i) $\left(M_{i}^{X}, M_{i}^{X^{\prime}}\right)$, (ii) $\left(M_{i}^{X}, M_{b}^{X^{\prime}}\right)$ and (iii) $\left(M_{b}^{X}, M_{b}^{X^{\prime}}\right)$ where $X \leq X^{\prime}$ in the lattice partial order (i.e., $\left.X^{\prime} \subseteq X\right)$. Each of the three cases splits further into two subcases, depending on whether or not the first stratum in the pair is $M_{i}$ or $M_{b}$, or is something else.

Case (i)a. $\left(M_{i}, M_{i}^{X^{\prime}}\right)$. We suppose $x_{j} \rightarrow y$, where $\left\{x_{j}\right\}$ is a sequence in $M_{i}, y \in$ $M_{i}^{X^{\prime}}$. Further, we suppose that the sequence of planes

$$
\tau_{j}=\operatorname{ker}\left(d\left(Q \mid M_{i}\right)_{x_{j}}\right) \subset T\left(D^{2 \ell} \times I\right)_{x_{j}}
$$

converges to a plane $\tau \subseteq T\left(D^{2 \ell} \times I\right)_{y}$. We consider $\kappa=\operatorname{ker}\left(d\left(Q \mid M_{i}^{X^{\prime}}\right)_{y}\right)$. Since $Q(x, t)=(0, t)$ for all $x \in M_{i}^{X^{\prime}}, \kappa=X^{\prime} \times\{0\} \subset \mathbb{C}^{\ell} \times \mathbb{R}=T\left(D^{2 \ell} \times I\right)_{y}$. We wish to show that $\kappa \subset \tau$.

Denote by $(\operatorname{grad} Q)_{x_{j}}$ the vector $\left(\frac{\partial Q}{\partial z_{i}}\right)_{x_{j}}$. Now $\tau_{j}=(\operatorname{grad} Q)_{x_{j}}^{\perp} \times\{0\} \subset \mathbb{C}^{\ell} \times \mathbb{R}$. Clearly $\tau=(\operatorname{grad} Q)_{y}^{\perp} \times\{0\} \subset \mathbb{C}^{\ell} \times \mathbb{R}$. A point $v \in \kappa=X^{\prime} \times\{0\}$ has the form $\left(v_{1}, \ldots, v_{\ell}, 0\right)$, with $\left(v_{1}, v_{2}, \ldots, v_{\ell}, \pi(y)\right) \in M_{i}^{X^{\prime}}$ and $Q\left(v_{1}, \ldots, v_{\ell}, \pi(y)\right)=0$. For $t$ fixed, $Q$ is a homogeneous function of the variables $z_{1}, \ldots, z_{\ell}$, of degree $n$. Thus one has the functional ("Euler") identity

$$
Q_{t}(z)=\sum_{i} \frac{z_{i}}{n} \frac{\partial Q_{t}}{\partial z_{i}}
$$

Thus,

$$
0=Q_{\pi(y)}(v)=\frac{1}{n} v \cdot(\operatorname{grad} Q)_{y}
$$

and so $v \in(\operatorname{grad} Q)_{y}^{\perp} \times\{0\}$. Thus $\kappa \subset \tau$ as desired.

The cases (ii)a and (iii)a are similar.

Case (i)b. $\left(M_{i}^{X}, M_{i}^{X^{\prime}}\right)$. Then

$$
\operatorname{ker}\left(d\left(Q \mid M_{i}^{X}\right)_{x_{i}}\right)=X \times\{0\} \subset\left(\mathbb{C}^{\ell} \times \mathbb{R}\right)_{x_{i}}=T\left(D^{2 \ell} \times I\right)_{x_{i}}
$$

certainly converges to $\tau=X \times\{0\} \subset T\left(D^{2 \ell} \times I\right)_{y}$. But $\operatorname{ker}\left(d\left(Q \mid M_{i}^{X^{\prime}}\right)_{y}\right)=$ $X^{\prime} \times\{0\} \subset \tau=X \times\{0\} \subset T\left(D^{2 \ell} \times I\right)_{y}$, so that $a_{Q}$ holds in this case.

The cases (ii)b and (iii)b are similar.

Thus $Q$ is a Thom mapping and the theorem follows by Thom's second isotopy lemma.

Corollary. Lattice-isotopic arrangements have diffeomorphic Milnor fibers.

Finally, we point out that Rybnikov [6] has exhibited arrangements with the same lattice and non-isomorphic fundamental group. Thus the requirement of a lattice isotopy in the above results is necessary.

\section{REFERENCES}

[1] M. Goresky and R. MacPherson, Stratified Morse Theory, Ergeb. Math. Grenzgeb. 3 (14), Springer, Berlin, 1988. MR 90d:57039

[2] J. Mather, Notes on topological stability, Harvard University, 1970, mimeo-graphed notes. 
[3] J. Milnor, Singular Points of Complex Hypersurfaces, Ann. Math. Studies 61 (1968), Princeton. MR 39:969

[4] P. Orlik and L. Solomon, Combinatorics and topology of complements of hyperplanes, Invent. Math. 56 (1980), 167-189. MR 81e:32015

[5] R. Randell, Lattice-isotopic arrangements are topologically isomorphic, Proc. Amer. Math. Soc. 107 (1989), 555-559. MR 90a:57032

[6] G. Rybnikov, On the fundamental group of a complex hyperplane arrangement, preprint, 1993.

[7] H. Whitney, Tangents to an analytic variety, Ann. of Math. 81 (1965), 469-549. MR 33:745

Department of Mathematics, University of Iowa, Iowa City, Iowa 52242-0001

E-mail address: randell@math.uiowa.edu 